\title{
The Prosthetic Generation Is all Around Us: Feelings and Emotions About Knee Replacement Surgery and Their Impact on Overall Sentiment: An Abstract
}

\author{
Christine Pitt, Amir Dabirian, Elsamari Botha, Jan Kietzmann, \\ and Hoda Diba
}

\begin{abstract}
In their attempts to reduce the uncertainty associated with knee replacement surgeries, patients turn to social media, where they commonly rely on the experiences expressed by other patients. In this study, we first employ IBM Watson to examine how patients talk about their emotions and express sentiment through their comments online. We then use a latent class cluster modelling procedure to segment these patients into distinct groups, according to their emotions (anger, disgust, fear, happiness, sadness and surprise), sentiment and their overall satisfaction with knee replacement surgery. Our findings show how qualitative online data can be transformed into quantitative insights regarding underlying market segments, which could then be targeted through different strategies by both marketers and healthcare practitioners.
\end{abstract}

References Available Upon Request

C. Pitt • A. Dabirian

Royal Institute of Technology (KTH), Stockholm, Sweden

e-mail: christinepitt91@gmail.com; adabirian@fullerton.edu

E. Botha

University of Cape Town, Cape Town, South Africa

e-mail: elsamari.botha@uct.ac.za

J. Kietzmann $(\bowtie)$

Simon Fraser University, Vancouver, Canada

e-mail: jkietzma@sfu.ca

H. Diba

Lulea University of Technology, Lulea, Sweden

e-mail: hoda007@yahoo.com 